\title{
Comunicación e interacción por el uso de dispositivos tecnológicos y redes sociales virtuales en estudiantes universitarios
}

\author{
Luis E Ruanoํ, Ernesto L Congote ${ }^{2}$, Andrés E Torres ${ }^{3}$
}

luiseruanoi@gmail.com, ernesto.congote@campusucc.edu.co, andrese.torresc@campusucc. edu.co

${ }^{1}$ Profesor Investigador, Universidad Cooperativa de Colombia, UCC, Programa de Psicología, Popayán, Colombia.

${ }^{2}$ Investigador, Universidad Cooperativa de Colombia, UCC, Programa de Psicología, Popayán, Colombia.

${ }^{3}$ Investigador, Universidad Cooperativa de Colombia, UCC, Programa de Psicología, Popayán, Colombia.

DOI: $10.17013 /$ risti.19.15-31

Resumen: El presente artículo describe tipologías de usuarios tecnológicos (nativo, inmigrante, reflexivo y escéptico), resultantes de la aplicación de la técnica de análisis sociológico del sistema de discursos desde el enfoque cualitativo, sobre un corpus documental compuesto por grupos de discusión. Se elige una muestra de estudiantes universitarios de dos programas académicos de la Universidad Cooperativa de Colombia Sede Popayán, con los cuales se realizan cuatro grupos de discusión hasta lograr la saturación discursiva; la inclusión de los sujetos al interior de la tipología se logra tras la definición de cuatro categorías de análisis (percepción, tipo de uso, frecuencia de uso y nivel de construcción o redefinición de la identidad), que permiten situar discursivamente al sujeto, frente a las formas de comunicación e interacción mediadas por dispositivos tecnológicos (computadoras, celulares, tabletas electrónicas) y redes sociales virtuales (Facebook, Twitter, YouTube y WhatsApp), considerando el impacto generado en su vida académica y personal.

Palabras-clave: Comunicación; interacción; identidad; redes sociales virtuales; TIC.

\section{Communication and interaction by the use of technological devices and virtual social media in university students}

Abstract: This article describes technology user's typologies (native, immigrant,
reflexive and skeptical) as a result of the speech's system sociological analysis
application, from the quantitative approach on a documentary corpus integrated
by discussion groups. A sample of students from two different programs from
Universidad Cooperativa de Colombia, Popayán headquarter is chosen, with
whom are performed four discussion groups until reaching discursive saturation;
the incorporation of the subjects to the typology is done after the four analysis
categories are defined (perception, kind of use, use frequency and level of identity
construction or identity redefinition) that allows to place discursively the subject in 
front of the diverse technological device mediated communication and interaction ways (computers, mobile phones, tablets) and virtual social media (Facebook, Twitter, YouTube and WhatsApp), considering the generated impact in their personal and academic lives.

Keywords: Communication, interaction, identity, virtual social media, information and communication technologies.

\section{Introducción}

El presente trabajo, hace parte del proyecto "Evolución del fenómeno de redes sociales y el acceso en movilidad: herramienta de comunicación y relación/interacción, en la ciudad de Popayán, Cauca y da continuidad al artículo "Dispositivos Tecnológicos: Comunicación e Interacción en un entorno Universitario", disponible las Actas del $5^{\circ}$ Congreso Ibero-Americano en Investigación Cualitativa, realizado en Porto Portugal, entre los días 12 y 14 de Julio.

La aparición de dispositivos tecnológicos de información (DT) como las computadoras, los teléfonos móviles y las tabletas electrónicas, han permitido la reconfiguración de las formas en que los seres humanos reciben, interpretan, administran y trasmiten la información; posibilitando el desarrollo de procesos de comunicación, interacción y de establecimiento de relaciones interpersonales mediados por el uso de la tecnología, donde la bidimensionalidad propuesta entre la realidad virtual (online) y la realidad no virtual (offline), afecta los comportamientos individuales y colectivos dentro y fuera de la red (Aguilar \& Said, 2010; Vázquez et al., 2016). Así, los fenómenos sociales se trasladaron a la virtualidad, sufriendo mutaciones que representan nuevas áreas de investigación (Aymerich, 2013) en las cuales, se contempla como fenómenos de estudio, los procesos relacionados con la adquisición de dispositivos, y la utilización de redes sociales y aplicaciones virtuales en las cuales los sujetos actúan, se relacionan y definen su identidad (Gonçalves et al, 2016).

Existe una tendencia a pensar que las nuevas formas de comunicación e interacción en la virtualidad podrían reemplazar formas tradicionales -como las cartas de amor y los periódicos escritos en papel--, no obstante, algunos académicos sostienen que más allá de reemplazarlas, la aparición de estas nuevas formas de comunicación desencadenan una relación de complementariedad (Azuela, 2011) en la que los usuarios pueden retomar aspectos online y offline de acuerdo a sus necesidades e intereses, de acuerdo al contexto y posibilidades de la interacción-comunicación. Más allá de esto, resulta difícil negar que "vivimos en una época en la que las relaciones interpersonales han evolucionado de tal manera que muchos encuentros, amistades y conversaciones tienen lugar a través de Internet" (Martínez, 2012, p. 23).

Si consideramos que Marshall McLuhan afirma que "el medio es el mensaje", es posible suponer que las interacciones y formas de comunicación en RSV en el plano virtual son distintas a las interacciones y comunicación en redes sociales en el plano no virtual, teniendo en cuenta que "la información transmitida a través de un medio no es tan relevante, por sí sola, sino que adquiere su poder transformador a través del medio y el contexto por el cual se difunde"(Aguilar, D; Said, 2010, p. 192). En este orden de ideas, "los espacios virtuales de socialización ofrecen nuevas instancias de comunicación, 
generación de nuevos códigos comunicativos y sistemas de significación. Así mismo, ofrecen la posibilidad al individuo, en tanto que sujeto, de convertirse en emisor y mensaje de sí mismo" (Aguilar, D; Said, 2010, p. 193)

El incremento del uso de DT, se asocia principalmente a cuatro factores: la aparición constante de campañas mediáticas y publicitarias que influyen sobre la percepción de necesidad e incitan la decisión de adquirir el dispositivo; los diferentes precios de acuerdo a las marcas y el tipo de tecnología, las facilidades de pago y la variedad de servicios que ofrecen; las ventajas percibidas por los mismos sujetos en el contacto cotidiano con los otros; y la posibilidad de descargar aplicaciones como Facebook, Twitter, YouTube y WhatsApp que permiten desarrollar todo tipo de actividades, ampliando el esquema de las interacciones y los canales de comunicación no virtuales del usuario, hacia el universo de las redes sociales virtuales. Es tan alta la popularidad de las RSV y tan fuerte el vínculo que mantienen con los DT, que la mayoría de dispositivos asocian en sus plataformas las principales RSV, incluso las ofertan preinstaladas, de esta manera, el más ajeno de los artilugios tecnológicos brinda alguna posibilidad de conectarse a las redes sociales.

Este incremento, sugiere el desarrollo de una sociedad con gran flujo de información, donde el conocimiento se construye y deconstruye constantemente, dando lugar a múltiples verdades, conocimientos y estilos de interacción desde los cuales los sujetos actúan y definen su identidad (Martínez, 2011). La tecnología ha promovido el surgimiento de una cultura nómada y global, con acceso a todo tipo de información proveniente de cualquier lugar del mundo, en constante crecimiento y de manera ilimitada. Tal como lo refiere Alarcón "en los últimos años, la utilización de Internet como canal global de comunicación ha estimulado a los individuos a cambiar sus maneras de interactuar entre sí, sus hábitos de ocio y sus formas de conocerse y relacionarse" (2012, p. 32), lo que ocasiona entre otras cosas, la naturalización del uso de internet y de las RSV para desarrollar actividades cotidianas.

Dicha construcción de significados individuales y colectivos en redes, se genera a través de múltiples encuentros discursivos y experiencias que tienen lugar en un plano bidimensional (de afectación mutua), donde los acontecimientos de la realidad virtual influyen sobre las percepciones y actitudes asumidas en la realidad no virtual, y a su vez los hechos trasladados desde la realidad no virtual hacia la virtualidad, generan interpretaciones y reacciones específicas en las redes. Según Caldevilla "de la interactividad propia del medio, nace la actual ventaja o desventaja de poder generar cambios de conducta, crear nuevos movimientos de opinión, promover manifestaciones, crear grupos de apoyo a causas concretas o conseguir crear una moda que genere el consumo de un determinado producto"(Cornejo, Tapia, 2011, p. 221) Lo que refuerza la idea de que la interacción y el lenguaje (comunicación) configuran la estructura de la personalidad de los sujetos, quienes, a partir de sus interacciones, modifican la estructura social del colectivo (Lewin, 1952)

Con el fin de comprender la dinámica generada por el uso RSV y DT, se analizan aquí, las percepciones de una muestra de 36 estudiantes, interpretando las formas de comunicación y de interacción comunes, y el impacto generado en su vida académica y personal. De este modo, se identifican similitudes y diferencias que permiten obtener tipologías específicas donde se sitúa discursivamente a los sujetos. Se elige una muestra de estudiantes de primero y último semestre de los programas académicos de Psicología 
e Ingeniería de Sistemas, de acuerdo al grado de familiaridad o cercanía que podrían tener con el tema, según el contexto educativo en el que se encuentran, su formación profesional y las prácticas cotidianas (informales) que desarrollan interactuando con personas que usan dispositivos tecnológicos y redes sociales. Tal como afirma Prensky "Los universitarios de hoy constituyen la primera generación formada en los nuevos avances tecnológicos, a los que se han acostumbrado por inmersión al encontrarse, desde siempre, rodeados de ordenadores, vídeos y videojuegos, música digital, telefonía móvil y otros entretenimientos y herramientas afines" (Cortés, 2016, p. 486).

La relevancia social de la investigación, se vincula con el reconocimiento de las formas de comunicación e interacción por el uso de DT y RSV en los escenarios educativos; a modo de identificar estilos de enseñanza, contenidos curriculares, fuentes y tipos de información, y posibilidades de adopción-creación de herramientas coherentes con las dinámicas actuales, para promover aprendizajes significativos en los estudiantes. La relevancia metodológica, hace referencia a la utilización de las técnicas cualitativas como los grupos de discusión y el análisis sociológico del sistema de discursos, cuyo principio y fin es la percepción subjetiva del participante, para obtener resultados válidos y confiables para la elaboración de teorías y propuestas metodológicas. Por último, la pertinencia del estudio radica en la presentación de una tipología de análisis de los usuarios-consumidores tecnológicos, que permitirá a los investigadores de las ciencias de la educación, las ciencias sociales y humanas, y las ciencias de la tecnología e informática, entre otras, reflexionar sobre estos fenómenos, generar otro tipo de análisis y aportar de manera crítica, nuevas respuestas e interrogantes al estudio de las RSV y el uso de DT.

\section{Aproximación teórica}

Bastardas, define la comunicación como "el intercambio de sentimientos, opiniones, o cualquier otro tipo de información mediante el habla, estructuras semióticas u otros tipos de señales" (2014, p. 78); esencialmente, la comunicación es un medio de satisfacción de necesidades. Según Martínez, "los distintos discursos que tienen lugar en nuestra sociedad están enmarcados por relaciones interpersonales entre los participantes en la interacción. En dichas interacciones los hablantes tienen a su disposición todas las opciones disponibles en el sistema de la lengua, y eligen el vocabulario y las estructuras gramaticales teniendo en cuenta su fin comunicativo" (Martínez, 2012, p. 109); siendo las interacciones entre al menos dos agentes que comparten un mismo repertorio de signos con reglas semióticas similares, las que permiten que exista el proceso comunicativo. Esencialmente, la información es incluida por el emisor en un paquete y es canalizada hacia el receptor a través del medio, quien es el encargado de decodificar el mensaje y proporcionar una respuesta (Thomson, 2008)

Según Fernández, "el escenario de la comunicación está caracterizado en la actualidad por cuatro elementos bien diferenciados: hiperconectividad, información social, deslocalización, y multipantalla, lo que implica una gran variedad en los dispositivos y en la forma de acceso a la información"(Fernández, 2015, p. 372) Para llegar a una comprensión de las formas de comunicación en este contexto, es necesario diferenciar la actividad informativa del acto comunicativo; "en el primero prevalece el carácter estático en el rol del emisor, mientras que el proceso comunicativo se caracteriza por la acción recíproca entre emisor y receptor al cambiar constantemente dichos roles" (Fernández, 2013, p. 522). 
En la actualidad, las formas de comunicación están mediadas por las Tecnologías de la información y la comunicación, TICs, los DT y las RSV. Las TIC, se conciben generalmente como el conjunto de avances tecnológicos desarrollados para gestionar información y enviarla de un lugar a otro, donde se desarrollan procesos de almacenamiento, recuperación y comunicación de la información, trabajando de manera interactiva e interconectada en torno a tres medios básicos: la informática, la microelectrónica y las telecomunicaciones (Cabero, 1991).

Por su parte, los Dispositivos Tecnológicos de Información (DT) son objetos que satisfacen necesidades de manera virtual y física a través de la tecnología; siendo tangibles (hardware) e intangibles (software), con la posibilidad de integrarse en las actividades de los individuos que requieran almacenar, procesar, interpretar, administrar y gestionar grandes cantidades de información. En la actualidad, estos dispositivos están asociados a Redes Sociales Virtuales, las cuales operan como sistemas de relación entre usuarios (Campos et al., 2015) que permiten administrar y generar contenidos, establecer y mantener relaciones a partir de la información contenida en los perfiles (Romero et al., 2011) abordar temas de interés, vivir fantasías y realizar transacciones (Casaló; Guinalíu, 2012) así como satisfacer necesidades personales, laborales, comerciales o académicas según el tipo de red y las posibilidades de interacción otorgadas a los usuarios.

Entre tanto, como afirman (Stornaiuolo y otros, 2013) las redes sociales pueden percibirse también, como un espacio orientado a la comunicación, que ofrece a los participantes nuevas oportunidades para el desarrollo de comunidad, en un espacio que proporciona múltiples vías para la construcción de puntos de vista compartidos mediante el uso de diversas herramientas semióticas. Al respecto, Canclini (1997) realiza una reflexión en torno al fenómeno de la comunicación, refiriendo cambios en el modo de consumo que han alterado las posibilidades y formas de ser ciudadano; afirmando, que los sujetos con acceso a determinado tipo de tecnologías desarrollan distintos modos de relación y estilos de vida frente aquellos que no lo tienen, lo que sugiere la existencia de una brecha que separa a unos de otros en el plano sociodemográfico y actitudinal.

Es importante resaltar, que el significado de los comportamientos de los usuarios en RSV, varía en función del contexto donde se produce la interacción (Martínez, 2012) y la interpretación del mensaje, quien a su vez es afectado por el medio en que se transmite (Moscoloni; Castro, 2010). Según Páramo y Burbano (2013), en las sociedades contemporáneas, las nuevas tecnologías de información y comunicación ejercen distintos tipos de influencias en los procesos que conllevan la socialización; dependiendo de la estructura de la comunicación social (Jones, 2005), y el constante dinamismo y actividad, que promueven la construcción y redefinición de la personalidad (Rueda, 2008).

En este punto, es posible afirmar, que el impacto generado por los DT y las RSV, no se reduce exclusivamente a la percepción de utilidad, donde existen necesidades específicas que se satisfacen por el uso de la tecnología; más allá de esto, concurre una compleja red formada por interacciones, normas y códigos semióticos profundos, que convocan procesos de construcción y redefinición de la identidad, estructuración de la realidad, sentimientos de pertenencia, aislamiento y malestar. "Las nuevas relaciones sociales, además de sistemas materiales y simbólicos, están integrados agentes y prácticas culturales, interacciones y comunicaciones, colectivos, instituciones y sistemas organizativos, una multiplicidad de contenidos y representaciones simbólicas, junto con 
valores, significados, interpretaciones y legitimaciones, entre otros" (Pineda, Meneses, \& Téllez, 2013). Considerando la velocidad de expansión y el grado de naturalización del uso de la tecnología para el desarrollo de actividades cotidianas, Lizarralde y López (2011) advierten sobre la forma en que la sociedad concibe una cultura inminentemente digital; lo que implica nuevas formas de comprender, interactuar y comunicar.

\section{Metodología}

Se toma como universo de investigación la Universidad Cooperativa de Colombia sede Popayán; específicamente a los estudiantes de Psicología e Ingeniería de Sistemas. El Estudio toma como base la técnica del grupo de discusión (Ibáñez, 1979). El Grupo de Discusión, herramienta fundamental en este trabajo, lo hemos definido como un "Instrumento de recogida de información discursiva, realizada en un entorno controlado, donde los componentes del grupo representan los distintos estratos de la estructura del objeto de investigación.” El grupo de discusión se fundamenta en la extracción de los significados, normalmente con un fuerte carácter social. Y que están estructurados de acuerdo a un orden o esquema de pensamiento. El grupo de discusión es una reconstrucción artificial, "en laboratorio", del discurso natural que podemos encontrar en un contexto estructural (Julia, Galindo, \& Galindo, 2014).

Los elementos extraídos de los grupos de discusión, son codificados y presentados como unidades temáticas o conceptuales que son utilizadas por el hablante. En las técnicas cualitativas, el investigador(a) tiene en consideración la construcción social de sentido (Julia et al., 2014). Así, en primer lugar, se plantearon los protocolos (grupos heterogéneos según casilleros tipológicos) y el modelo de moderación. Los mismos, fueron grabados en audio y video y luego transcritos textualmente, para ser analizados a través del software de análisis cualitativo Atlas.Ti, a partir de pautas propuestas por la teoría fundamentada, identificando las categorías iniciales a través de la codificación abierta (Strauss; Corbin, 2002).

Se crearon las tipologías de los consumidores tecnológicos a partir de cuatro variables: percepción del dispositivo tecnológico, uso que le dan, frecuencia de uso y construcción y redefinición de la identidad del sujeto por el uso de los mismos. Dentro de los grupos, se pretendió encontrar posturas discursivas que representaran de mejor forma, o fueran más ilustrativas para la categoría. Se intenta el desarrollo de una estructura de categorías y su interpretación en un contexto no necesariamente replicable, pero si con posibilidad de transferirse a otros contextos (Costa, Mónica, \& Paulo, 2015). En definitiva, se realizaron cuatro grupos a partir de los cuales se obtuvo la saturación discursiva (Krueguer, 1991), conformados por informantes que cumplieron básicamente la función de ilustrar cada una de las categorías de los perfiles, acotar y ordenar la interpretación.

En un segundo momento del análisis de los datos cualitativos, se implementó el análisis sociológico del sistema de discursos (Gutierrez del Alamo, 2009), en una muestra de tipo estructural que representa las distintas posiciones discursivas, mas no la generalización de resultados. Considerando las ventajas que ofrece el grupo de discusión para explorar el contexto de las nuevas formas de relaciones sociales (o virtuales), este análisis parte de la descripción biográfica de perfiles, pasa por un análisis semiótico-estructuralista, complementándose con un análisis de los contenidos manifiestos y latentes, hasta llegar a una dimensión más bien pragmática o interpretativa del contexto. 


\section{Modelo Central}

El modelo central expone cuatro categorías obtenidas en la fase final de la investigación: Nativos tecnológicos, Inmigrantes tecnológicos, Reflexivos tecnológicos y Escépticos tecnológicos, estas representan las posiciones discursivas relativas a cada sujeto, permitiendo su identificación en un orden simbólico individual y colectivo.

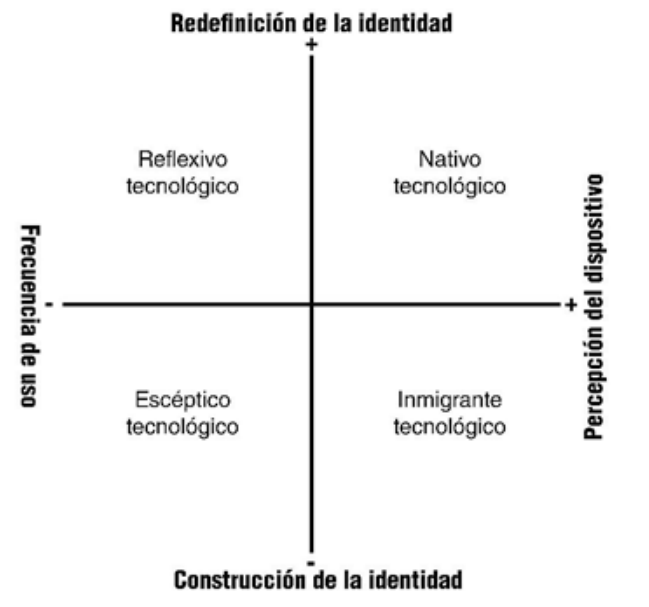

Figura 1 - Tipología de consumidores tecnológicos, cada tipo se correlaciona con uno o más ejes

\section{Hipótesis}

Se plantean cuatro supuestos de orden inductivo, considerando las actitudes posibles de los sujetos de acuerdo a las categorías que los representan:

i. Los Nativos tecnológicos, construyen su identidad a partir del uso de dispositivos y la interacción en redes, descuidando aspectos de la interacción no virtual.

ii. Los Inmigrantes tecnológicos, sobrevaloran la importancia de los dispositivos y las relaciones en red, otorgándoles un lugar central en sus vidas.

iii. Los Reflexivos tecnológicos, tienden a estar bien informados sobre la utilidad de los dispositivos y RSV, evitando hacer uso inadecuados de los mismos.

iv. Los Escépticos tecnológicos, son proclives a generar percepciones, creencias y expectativas negativas respecto al uso de los dispositivos, para justificar la decisión de no usarlos.

\section{Análisis}

El análisis parte desde una aproximación o retrato sociológico de cada uno de los perfiles, como una forma de ofrecer los primeros antecedentes contextuales. 


\subsection{Nativos tecnológicos.}

Su característica principal es la cercanía que mantienen con los DT y las RSV; interactúan con mayor facilidad en la realidad online que en la realidad offline; se muestran confiados desarrollando actividades en redes y les atrae establecer numerosas relaciones con amigos, contactos o seguidores al interior de las mismas; la mayoría de ellos crecieron a la par con los DT contemporáneos y tuvieron acceso a RSV desde temprana edad, lo que les ha permitido adquirir habilidades y capacidades de aprendizaje distintas a quienes no tuvieron dicho acceso; perciben el uso de estos medios como algo normal, por lo que se sienten cómodos accediendo a ellos de manera casi continua para realizar todo tipo de actividades diarias; suelen experimentar incomodidad, angustia o malestar cuando se ven obligados a relacionarse sin hacer uso de los DT.

El fragmento discursivo seleccionado para representar esta tipología, se obtuvo de un estudiante de primer semestre del programa de Psicología: "El celular es lo primordial o la herramienta de comunicación más importante; una vez me castigaron - se refiere a sus padres-- y era horrible, estar sin el celular. Ahora todo se puede hacer desde el cel -refiriéndose al celular--, puedes ver que aparecen emoticones y uno se da cuenta que hasta eso va evolucionando; ya no hay necesidad de llamarse, puedes solo estar conectado todo el día al Facebook. Allí están los medios para estar en contacto con todos, entonces las notas de voz también juegan un papel importante, si ya estoy aburrida de escribir, ya duele-se refiere a sus dedos---- o para darse a entender mejor. También para la universidad, pueden las tecnologías ayudar para una exposición, no sé qué haríamos sin ellos."

\subsection{Inmigrantes tecnológicos.}

Su principal característica es el evidente interés por adquirir DT y participar en RSV; conceden igual importancia a los acontecimientos ocurridos en la realidad online y a los acontecimientos ocurridos en la realidad offline; perciben mayores ventajas que desventajas; integran los DT y redes a sus necesidades cotidianas y las usan como una forma de mantenerse visibles ante la sociedad; son capaces de discernir y establecer límites en relación a la frecuencia de uso de las RSV; pueden tener dificultades para comprender los significados de ciertos acontecimientos que ocurren en la virtualidad, cuando estos resultan extraños o ajenos a su experiencia en el plano no virtual. Son sujetos que nacieron en una época previa a la creación de los DT contemporáneos, por lo que vivenciaron el inicio y evolución de las formas actuales de interacción en redes; intentan mantenerse a la vanguardia de la tecnología y frecuentan RSV de acuerdo a la utilidad percibida, por lo que hay una tendencia a perder el interés y abandonar estos medios cuando perciben que sus expectativas no son satisfechas, o experimentan durante la monotonía por periodos prolongados de tiempo.

El fragmento discursivo seleccionado para representar esta tipología, se obtuvo de un estudiante de décimo semestre de Psicología: "Si hablamos de tecnología pues uno puede decir que se utiliza para investigaciones o para otras cosas, pero como ya va encaminado con la comunicación, yo creo que se utiliza ahora más la tecnología para comunicarse que hacer la visita o estar así presenciales, a veces no es tanto pereza sino comodidad, uno se puede comunicar fácil. Hay cosas que te obligan a entrar en el uso de los aparatos, el grupo en el WhatsApp, la familia en el WhatsApp... eso ya se 
volvió algo fundamental, aparte de que es adictivo, es necesario, imuy necesario! La desventaja es que no se sabe interpretar lo que la persona está diciendo, uno puede leer a su forma, a su manera y puede malinterpretar lo que escriben, es así como en clase... (Risas) o en práctica: cuando uno está haciendo o lo coge el celular, pero no es bueno, es muy incómodo salir con una persona y que esté pegada a eso, se dañan los momentos."

\subsection{Reflexivo tecnológico.}

Su principal característica es la prevención y el establecimiento de límites relativamente estrictos frente al uso de DT y RSV; conceden mayor importancia a las interacciones producidas en el plano offline que aquellas ocurridas en el plano online; son personas que crecieron al margen de los DT contemporáneos o hicieron uso restringido de ellos; son capaces de analizar las ventajas y desventajas que conlleva el uso de los DT y RSV evaluando los tipos de acceso y frecuencia de uso, así como la seguridad y privacidad al interior de los mismos; generalmente usan sólo aquella tecnología que les ayuda a satisfacer necesidades específicas a nivel laboral, académico o relacional, evitando utilizarlas para el entretenimiento o en los momentos de ocio; su principal argumento es que son las personas quienes usan y controlan los DT y no los DT quienes los controlan a ellos.

El fragmento discursivo seleccionado para representar esta tipología, se obtuvo de un estudiante de primer semestre del programa de ingeniería de sistemas: "Creo que la tecnología no es mala por sí misma, lo malo es el uso que le demos, lastimosamente las tecnologías que tenemos ahorita --silencio prolongado-- han impactado mucho la forma de relacionarnos y de cómo tal vez hacemos las cosas, pero la parte que realmente se afecta es la parte humana. Sería bueno hacer conciencia social para que las personas no desperdicien su tiempo, uno le puede sacar mucho provecho a muchas cosas de la tecnología, pero se pierden otras. Por ejemplo, los estudiantes casi ya no tenemos cuadernos porque todo lo hacemos por medios digitales, y estos no son del todo seguros."

\subsection{Escépticos tecnológicos.}

Su principal característica es la abstención del uso de DT y RSV; poseen creencias negativas, estrictas y rígidas validadas por la experiencia o la observación; prefieren emplear medios tradicionales o alternativos de interacción y comunicación como las llamadas telefónicas, el contacto cara a cara y las cartas escritas en papel; sobreestiman la realidad no virtual y en la mayoría de los casos ni siquiera se permiten conocer más acerca de la virtualidad; poseen poca capacidad de adaptación al cambio; pueden llegar a sentirse excluidos, invisibles o ajenos de los acontecimientos sociales. Pueden llegar a tener DT y RSV por algún tipo de presión social, sin que esto signifique abandonar su percepción de que la época anterior a la creación de dichos medios era mejor.

El fragmento discursivo seleccionado para representar esta tipología, se obtuvo de un estudiante de primer semestre del programa de Psicología: "Antiguamente no existía nada de eso y éramos felices --risas-- y tú veías entonces, si era un fin de semana o un viernes, estaban -se refiere a las personas-- en los parques o en las zonas comunes, en los barrios, comentándose qué les había pasado en el transcurso de la semana; tenías tu álbum fotográfico impreso en tu casa y tú cuando visitaban tu casa que eran conocidos, allegados tuyos, tú sacabas tu álbum y lo mostrabas. Ahora de forma digital ya no necesitas que la persona venga hasta ti para tú mostrarle algo que es 
significativo, sino que ya desde la distancia, tú le das acceso a esa información. En las redes sociales la mayoría de los niños o las niñas, adolescentes, lo toman como un medio de exhibición sexual: exhibirse los cuerpos, mantener el chisme... difusión, ventilación de la vida privada. Yo diría que eso es lo que está haciendo más que todo la juventud, con la tecnología. Yo no tengo ni siquiera WhastApp instalado en este celular; como yo no estoy cerca a mi familia, utilizo sólo para llamadas y no reviso ni mi correo de la universidad."

\section{Pares Sémicos y Ejes Estructurantes}

Se ubicó en la primera parte de la lista a modo de referencia, el par Nativo tecnológico Escéptico tecnológico, por ser estos perfiles los que se contraponen de forma más tajante en el modelo central. Así, el resto de oposiciones creadas a partir de esta distinción inicial, formaran parte de las propiedades que caracterizan a estos perfiles.

\begin{tabular}{|c|c|c|}
\hline Ejes & Nativo Tecnológico & Escéptico Tecnológico \\
\hline \multirow[t]{2}{*}{$\begin{array}{l}\text { Percepción del } \\
\text { dispositivo }\end{array}$} & $\begin{array}{l}\text { Consideran que disminuyen el esfuerzo } \\
\text { y hacen la vida más fácil, permiten } \\
\text { nuevas posibilidades de comunicación e } \\
\text { interacción entre las personas. }\end{array}$ & $\begin{array}{l}\text { Creen que limitan aspectos } \\
\text { fundamentales de la comunicación y } \\
\text { la interacción. }\end{array}$ \\
\hline & $\begin{array}{l}\text { Se enfocan más en las ventajas que en las } \\
\text { desventajas }\end{array}$ & $\begin{array}{l}\text { Se enfocan más en las desventajas } \\
\text { que en las ventajas }\end{array}$ \\
\hline Usos & $\begin{array}{l}\text { Los usan tanto para comunicarse e } \\
\text { interactuar como para divertirse y } \\
\text { distraerse en su tiempo libre, manejan } \\
\text { muchas redes sociales y lugares virtuales. }\end{array}$ & $\begin{array}{l}\text { Los usan exclusivamente para } \\
\text { trabajar o realizar actividades } \\
\text { específicas, solo tienen correo } \\
\text { institucional o cosas obligatorias. }\end{array}$ \\
\hline Frecuencia de uso & $\begin{array}{l}\text { Muy alta (se utiliza en momentos donde } \\
\text { interrumpe u obstaculiza la comunicación } \\
\text { e interacción presencial con otros) }\end{array}$ & $\begin{array}{l}\text { Muy baja o nula (Cuando es } \\
\text { obligatorio, donde no interrumpe } \\
\text { la comunicación e interacción } \\
\text { presencial con otros) }\end{array}$ \\
\hline $\begin{array}{l}\text { Construcción o } \\
\text { reconstrucción de } \\
\text { la identidad }\end{array}$ & $\begin{array}{l}\text { Fuerte identificación con el dispositivo } \\
\text { tecnológico, revisten el objeto de } \\
\text { significado otorgándole un rol activo en sus } \\
\text { vidas, interiorizan el objeto y lo reconocen } \\
\text { como una extensión de sí mismos, sienten } \\
\text { incomodidad o malestar por la ausencia } \\
\text { del objeto y experimentan angustia } \\
\text { por la pérdida definitiva del mismo. } \\
\text { En momentos de ocupación laboral o } \\
\text { académica, obstaculiza dichas actividades }\end{array}$ & $\begin{array}{l}\text { Poca identificación con el dispositivo } \\
\text { tecnológico, representa un rol } \\
\text { pasivo en sus vidas, elaboran una } \\
\text { diferenciación clara del objeto } \\
\text { respecto a ellos mismos, pueden } \\
\text { experimentar incomodidad con } \\
\text { el uso del dispositivo. Lo utilizan } \\
\text { solo cuando es obligatorio y no } \\
\text { obstaculiza actividades laborales o } \\
\text { académicas. }\end{array}$ \\
\hline
\end{tabular}

Tabla 1. - Pares Sémicos y Ejes Estructurantes

\section{Oposiciones Estructurantes}

Se han ubicado en la primera parte de la lista los cuatro tipos de consumidores tecnológicos con sus respectivas características, en relación a las variables encontradas en los grupos de discusión. Es aquí donde se puede observar con mayor precisión las características fundamentales de cada tipología, identificando similitudes y diferencias. 


\begin{tabular}{lllll}
\hline Ejes & Nativo Tecnológico & $\begin{array}{l}\text { Inmigrante } \\
\text { Tecnológico }\end{array}$ & $\begin{array}{l}\text { Reflexivo } \\
\text { Tecnológico }\end{array}$ & $\begin{array}{l}\text { Escéptico } \\
\text { Tecnológico }\end{array}$ \\
\hline $\begin{array}{l}\text { Percepción del } \\
\text { dispositivo }\end{array}$ & Útil e indispensable & $\begin{array}{l}\text { Necesario en casos } \\
\text { concretos. }\end{array}$ & $\begin{array}{l}\text { Útil, no } \\
\text { indispensable }\end{array}$ & Innecesario \\
\hline Usos & $\begin{array}{l}\text { Entretenimiento, } \\
\text { comunicación, } \\
\text { interacción, } \\
\text { aprendizaje y trabajo. } \\
\text { Necesidad. }\end{array}$ & $\begin{array}{l}\text { Trabajo, } \\
\text { aprendizaje, ocio, } \\
\text { comunicación, } \\
\text { interacción. } \\
\text { Comodidad. }\end{array}$ & $\begin{array}{l}\text { Trabajo, } \\
\text { aprendizaje, } \\
\text { comunicación. } \\
\text { Utilidad }\end{array}$ & $\begin{array}{l}\text { Trabajo, } \\
\text { comunicación. } \\
\text { Obligación. }\end{array}$ \\
\hline $\begin{array}{l}\text { Frecuencia de uso } \\
\text { Construcción o } \\
\text { redefinición de la } \\
\text { identidad }\end{array}$ & $\begin{array}{l}\text { Muy alta } \\
\text { Identificación asociada } \\
\text { interiorización del } \\
\text { objeto. }\end{array}$ & $\begin{array}{l}\text { Identificación } \\
\text { asociada a la } \\
\text { comodidad con } \\
\text { diferenciación } \\
\text { difusa del objeto. }\end{array}$ & $\begin{array}{l}\text { Alta - Media } \\
\text { asociada al } \\
\text { diferenciación del } \\
\text { objeto. }\end{array}$ & $\begin{array}{l}\text { Media - Baja } \\
\text { rechazo del } \\
\text { objeto. }\end{array}$ \\
\hline
\end{tabular}

Tabla 2. - Oposiciones Estructurantes

\section{Matriz de Relaciones Estructurantes}

El Reflexivo y el Nativo, comparten una tendencia a redefinir su identidad, a diferencia del Escéptico e Inmigrante, que mantienen cierta estabilidad de esta, debido a una construcción previa, estructurada a través de la interacción con objetos diferentes a las tecnologías contemporáneas. El Reflexivo, comparte con el Escéptico la tendencia a considerar las ventajas y desventajas y evaluar el nivel de satisfacción o insatisfacción por el uso de DT y RSV, mientras el Nativo y el Inmigrante comparten percepciones favorables de los mismos, que los lleva a mantener una alta frecuencia de uso/consumo.

La migración de los sujetos de una tipología a otra, ocurre de la siguiente manera: a) el Nativo, puede convertirse en reflexivo al experimentar situaciones desagradables o identificar desventajas por el uso de DT y RSV, asumiendo una postura racional y crítica; b) el Reflexivo puede convertirse en Inmigrante, al acentuar o encontrar nuevas ventajas en el uso de DT y RSV, asumiendo una postura más adaptativa y centrada en la utilidad; un Reflexivo también puede convertirse en Escéptico, si experimenta insatisfacción por el uso de DT y RSV, asumiendo una postura de abstención; c) el Inmigrante puede convertirse en Reflexivo, al establecer límites o regular el consumo de DT y RSV, asumiendo una postura racional y crítica; por último, d) el Escéptico puede convertirse en Reflexivo al sentir satisfechas sus necesidades de comunicación y de interacción por medio de DT y RSV, asumiendo una postura racional y crítica.

Aunque los sujetos pertenecientes a la tipología Nativo tecnológico pueden migrar a cualquier otra tipología, ninguno de los sujetos pertenecientes a otra tipología puede llevar a ser Nativo por el factor de la edad. En este sentido (Jiménez, Rebollo, García, \& Buzón, 2015) describe una "brecha de capital social en función de la edad, encontrando que la red social de las personas mayores es más reducida, aunque tiende a incluir personas de edades más diversas; también encuentran que los adolescentes hacen más 


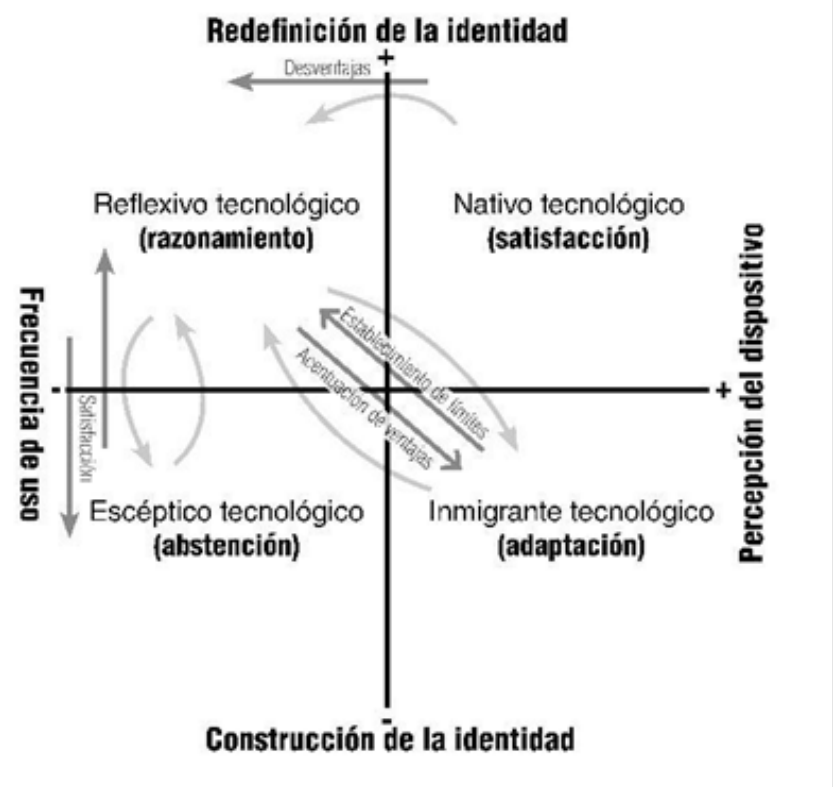

Figura 2 - El esquema presenta las posiciones discursivas que identifican a los sujetos al interior de las tipologías

uso de diferentes medios y más autorreferencias cuando se describen a sí mismos que las personas mayores."

\section{Discusión Final}

La creación de tipologías o categorías de análisis sobre el consumo de dispositivos tecnológicos y redes sociales tiene precedentes significativos; Prensky, citado por (Campos et al., 2015) define dos tipologías de usuarios: los llamados Nativos Digitales, que nacieron en el marco de la sociedad de la información, y para quienes el empleo regular de estos sistemas interactivos de comunicación les resulta normal, y los Migrantes Digitales, en comparación con la generación predecesora, que vieron surgir y consolidarse a las TIC en su vida cotidiana, por lo que tuvieron que aprender a usarlas conforme se desarrollaban.

Posteriormente, Alarcón, citado por (Azuela, 2011) realiza una clasificación preliminar de los usuarios de redes sociales (RS) en España generando cuatro tipologías: Usuario introvertido. Integrado principalmente por hombres mayores de 45 años, su frecuencia de uso no va más allá de una hora a la semana, utilizan las RS para enviar mensajes privados; Usuario novel. Conformado principalmente por mujeres menores de 29 años, su frecuencia de uso es de 1 a 5 horas por semana, utilizan las RS para compartir fotos, enviar mensajes públicos y privados y comentar lo que dicen o hacen otras personas; Usuario versátil. integrado por hombres de entre 36 y 44 años que se conectan al menos una vez al día empleando hasta 5 horas diarias, suelen realizar la mayoría de las actividades del 
usuario novel, con la característica de que tienen perfiles en más de una red social; Usuario experto-comunicador. Conformado por mujeres de entre 25 y 35 años que se conectan a las $\mathrm{RS}$ varias veces, y hasta por más de 5 horas al día, son los usuarios más activos, además de las actividades anteriormente mencionadas, comentan anuncios y publicidad.

La similitud de las tipologías obtenidas en esta investigación, con las tipologías descubiertas por otros autores, es notoria, lo que sugiere cierto grado de confiabilidad de los resultados, aunque por el tipo de investigación, no se pretenda la generalización o replicación de estos - característica del enfoque cuantitativo.

Como lo refiere (Alarcón \& Lorenzo, 2012) la mayoría de los usuarios, con bastante frecuencia "mandan mensajes privados dentro de la red, comparten o cuelgan fotos en el espacio, comentan lo que dicen, hacen o las fotos de otros contactos, se informan de las cosas de su interés, mandan mensajes públicos normalmente publicados en el muro propio o en el de otros compañeros, etiquetan amigos en las fotos, curiosean en el perfil de otros usuarios y actualizan el perfil"; sin embargo, los intereses y motivaciones subyacentes, permanecen relativamente ocultos, y responden a posiciones discursivas diferenciadas, que permiten reconocer al sujeto y ubicarlo en determinada tipología. En este sentido, la comunicación y la interacción atraviesan el análisis de la subjetividad de los participantes, teniendo en cuenta que, a partir de estos, "en las redes virtuales se transforma la realidad, se transforman las relaciones y se transforma el sujeto" (Ruiz, 2015, p. 155).

La percepción de los nativos tecnológicos acerca de los DT y las RSV es favorable, ocupan un lugar importante en sus vidas, principalmente para socializar en la red. El entretenimiento en momentos de ocio son motivos usuales para acceder a ellos, los usos giran en torno a la curiosidad, el establecimiento de relaciones con gran número de contactos y la conformación de comunidades virtuales. Al respecto, como le refiere (Suriá \& Raquel, 2012, p. 18) "cabría la posibilidad de que el establecimiento de relaciones con desconocidos a través de estos espacios online, de algún modo, pudiera resultar enriquecedor no sólo por ampliar la red social sino porque al fomentar la interacción social facilitara el desarrollo de habilidades sociales de la persona.”

Las características de estos sujetos, concuerdan con los resultados de otras investigaciones que sugieren que "los jóvenes las han incorporado (las redes sociales virtuales) plenamente en sus vidas. Se han convertido en un espacio idóneo para intercambiar información y conocimiento de una forma rápida, sencilla y cómoda.”

Los inmigrantes tecnológicos, mantienen una actitud de adaptación, asociada con su percepción de utilidad. Conciben el uso de DT y RSV desde su experiencia no virtual, desde la cual intentan integrar y generar nuevos usos a sus prácticas cotidianas; utilizan las RSV para curiosear, entretenerse y socializar, pero también para estar informados, construir aprendizajes y mantener relaciones no virtuales en red. Esto concuerda con lo expuesto por (Zornoza, Orengo, \& Peiró, 2008) quien afirma que "las aproximaciones más recientes sobre los efectos de las TICs (McGrath \& Hollingshead, 1993; Walther, 1995) sugieren que con el tiempo los grupos adquieren experiencia en el uso del medio de comunicación con el que tienen que interactuar y pueden llegar a desarrollar nuevas estrategias para comunicarse y realizar la tarea."

Para los reflexivos tecnológicos las TIC, los DT y las RSV son medios útiles más no indispensables, por lo que pueden interactuar y comunicarse sin problemas dentro 
y fuera de la red; la frecuencia de uso depende en gran medida de la duración de la tarea que realicen, y los usos se enfocan principalmente en la actualización personal, la generación de nuevos aprendizajes y la visibilización de sus perfiles y actividades en red para obtener ventajas académicas o laborales. Esta posición discursiva se enmarca en la afirmación de (Cautín, 2015, p. 74) que sugiere que "en una red comunicativa virtual prima la relación que los sujetos sostienen fuera de internet (amigos cercanos, compañeros de universidad), la que se traspasaría a su relación virtual y le restaría importancia a los grados de poder virtual que poseerían los sujetos en la plataforma estudiada.”

La posición discursiva delos escépticos, se desarrolla en torno ala prevención y evitación de los medios tecnológicos, prefiriendo formas no virtuales para comunicarse e interactuar; refieren la seguridad y la privacidad como las principales áreas que se ven vulneradas por el uso de la tecnología; su frecuencia de uso y participación en redes llega a ser mínima o inexistente. Contrario a lo que se puede pensar, aún existen millones de personas en el mundo que no hacen uso de las TIC, los DT y las RSV contemporáneas; bien sea por prohibiciones internas en sus países, la brecha digital, dificultades económicas para la adquisición o, en el caso particular, preferencias/elecciones personales que refuerzan la decisión de no usarlas. Frente a la posición discursiva de los escépticos tecnológicos, existe evidencia que valida hasta cierto punto sus creencias y percepciones sobre la interacción en red; en este sentido, Bauman, citado por (Cornejo \& Tapia, 2011, p. 109) "enfatiza la fragilidad de los vínculos, siendo ésa fragilidad la que inspira sentimientos de inseguridad y deseos conflictivos de estrechar lazos, pero al mismo tiempo, éstos deben ser endebles para poder desanudarlos. (...) prima la desconfianza de una relación permanente, por temor a la carga y tensiones que pueda implicar, frente a lo cual no se significan capaces ni deseosos de soportar, ya que limitarían la libertad que necesitan.”

La relación que los usuarios identificados en las diferentes tipologías desarrollan al interior de las redes, se establece a partir de un componente ideológico, representado por la información almacenada en los perfiles/cuentas, que contienen fragmentos de la subjetividad e identidad virtual del sujeto; en términos generales, las interacciones entre usuarios -independientemente de la tipología a la que pertenezcan-- se realizan con base en parámetros de exclusividad y selectividad definidos por cada usuario, así como la posibilidad de los mismos para comunicar sólo aquello que desean, cuando lo desean; esto incluye proyectar una imagen social que puede corresponder o no a las características reales de los sujetos en el plano offline. De acuerdo con (Andrade \& Campo, 2014) "en la web las personas participan en comunidades virtuales, no sólo por una razón de identidad colectiva, sino por una reciprocidad ideológica."

Durante el transcurso de la investigación, se observó una tendencia en los usuarios tecnológicos, a referir acontecimientos y significados sociales que resultaban extraños para los no usuarios; por lo que se concluyó que el uso de DT y la participación en RSV, contribuyen al establecimiento de sentimientos de pertenencia e identidad entre los usuarios, diferenciándolos de los no usuarios. (Cort et al., 2015) "en la interacción social --el contacto con otras personas y la participación conjunta en actividades diversas - se entablan importantes procesos, como la socialización, la integración grupal y la construcción de la identidad." En este sentido, se hizo evidente, que los sujetos que no tenían acceso a los dispositivos y redes empleados por la mayoría, quedaban parcialmente excluidos de los acontecimientos y experiencias sostenidas por este medio, 
lo que ocasionaba desconocimiento de la información, disminución de la comunicación y restricción de las interacciones sociales. Es posible suponer, que la definición de la identidad de estos sujetos no usuarios se verá afectada por este fenómeno, teniendo en cuenta que "el simple reconocimiento de la existencia de un usuario es el más básico, pero también uno de los más importantes aspectos de la construcción social de la identidad." (Ortigosa \& Ibáñez, 2006)

En cuanto a la constitución de la identidad, se advierte una tendencia en los nativos tecnológicos a concebir los DT desde los que el efectúan sus actividades comunicativas y de interacción, como una prolongación de sí mismos; en este sentido, su identidad es susceptible de redefinirse en la medida en que cambian las posibilidades de comunicación/interacción por la evolución del dispositivo. Para ellos, "la tecnología no es solo mediadora, sino constructora de lo social” (Barrios, 2009).

Los inmigrantes, reflexivos y escépticos tecnológicos por otro lado, asumen una postura más crítica del uso de los DT y las RSV, generada por vivenciar el surgimiento y evolución de estos medios en una etapa posterior a la construcción de su identidad. Si bien la identidad varía de algún modo a lo largo del tiempo --de acuerdo a las experiencias y aprendizajes subjetivos, así como a los acontecimientos e influencias sociales--, es más probable que estos sujetos adapten los dispositivos a sus necesidades, en lugar de percibirlos como una necesidad en sí mismos.

A modo de reflexión, se plantea que el estudio de las formas de comunicación e interacción mediadas por dispositivos tecnológicos y redes sociales virtuales, requiere profundizar en otras áreas de su estructura para visibilizar el alcance de la inclusión de este tipo de tecnologías en las diferentes esferas de la sociedad.

\section{Agradecimientos}

A la Universidad Cooperativa de Colombia, sede Popayán, en particular al programa de Psicología, por la gestión realizada para la presentación y publicación de este trabajo. De igual manera, a los coordinadores del programa de Psicología e Ingeniería de Sistemas, por su apoyo para la aplicación de la técnica de los grupos de discusión.

\section{Referencias Bibliográficas}

Aguilar, D., \& Said, E. (2010). Identidad y Subjetividad en las redes sociales: Caso de Facebook. Zona Próxima, 1, 190-207.

Alarcón, M., \& Lorenzo, C. (2012). Diferencias entre usuarios y no usuarios de redes sociales.Enl@ce: Revista Venezolana de Información, Tecnología Y Conocimiento, 9(2), 31-49.

Andrade, J., \& Campo, R. (2014). Google: Análisis ideológico de las redes sociales en Internet. Quórum, 11(1), 96-111.

Aymerich, L. (2013). La realidad virtual como herramienta de estudio de fenómenos psicológicos y sociales. Revista, Orbis Ciencias, Científica, 8(24), 102-115.

Azuela, J. I. (2011). Redes sociales en México, una reflexión. CienciaUAT, 5(4), 29-33. 
Campos, C., Leticia, R., González, Z., Domínguez, M., Leticia, R., \& Campos, C. (2015). El estudio de los hábitos de conexión en redes sociales virtuales , por medio de la minería de datos.

Cautín, V. (2015). Poder virtual y formas de tratamiento en el discurso mediado por computadora: exploración en una red comunicativa virtual. Forma Y Función, 28(1), 55-78. http://doi.org/10.15446/fyf.v28n1.51971

Cornejo, M., \& Tapia, M. L. (2011). Redes sociales y relaciones interpersonales en internet. Fundamentos En Humanidades, 12(24), 219-229.

Cort, L., Alfredo, C., Gonz, Z., Men, H., Jos, P., \& Herrera, C. (2015). El estudio de los hábitos de conexión en redes sociales virtuales, por medio de la minería de datos. Innovación Educativa, 15(68), 99-114.

Cortés, R. (2016). Consumo de medios noticiosos en redes sociales virtuales ( RSV ) entre estudiantes universitarios Consumption of News Media in Virtual Social Networks (RSV ) Among College Students. Razón Y Palabra, 20(93), 483-496.

Costa, A. P., Mónica, B., \& Paulo, L. (2015). Investigação através do Desenvolvimento : Quando as Palavras "Contam " Research through Development: When Words " Count .” Revista Iberica de Sistemas Y Tecnologias de informaciónRISTI, 1(2007), 3-6. http://doi.org/10.17013/risti.e4.vii-x

Fernández, A. (2015). las generaciones más jóvenes . El Movimiento 15M y el Umbrella Movement. El Profesional de La Información, 24(4), 371-379.

Fernández Sánchez, N. (2013). Trastornos de conducta y redes sociales en Internet. Salud Mental, 36(6), 521-527.

Gonçalves, R., Martins, J., \& Rocha, Á. (2016). Internet e redes sociais como instrumentos potenciadores de negócio. RISTI-Revista Ibérica de Sistemas e Tecnologias de Informação, (18), 09-11.

Jiménez, R., Rebollo, M. Á., García, R., \& Buzón, O. (2015). Motivos de uso de las redes sociales virtuales: Análisis de perfiles de mujeres rurales. RELIEVE. Revista Electrónica de Investigación Y Evaluación Educativa, 21(1), 1-17. http://doi. org/10.7203/relieve.21.1.5153

Julia, D. C., Galindo, P. V., \& Galindo, M. P. (2014). Grupos de Discusión y HJ-Biplot : Una Nueva Forma de Análisis Textual. RISTI - Revista Ibérica de Sistemas e Tecnologias de Informação, 2014(E2), 19-35. http://doi.org/10.17013/risti.e2.19-35

Luis, V., Casaló, L. V, Flavián, C., \& Guinalíu, M. (2012). Redes sociales virtuales desarrolladas por organizaciones empresariales : antecedentes de la intención de participación del consumidor. Cuadernos de Economía Y Dirección de La Empresa, 15(1), 42-51. http://doi.org/10.1016/j.cede.2011.06.003

Martínez, M. (2012). Aproximación a la interacción virtual : el caso de la red social Badoo. Palabra Clave, 15(1), 107-127.

Ortigosa, A., \& Ibáñez, A. (2006). Comunicación en Internet: constructivismo social e identidad virtual. Comunicar, (27), 179-186. 
Pineda, E; Meneses, T; Téllez, F. (2013). Análisis de redes sociales y comunidades virtuales de aprendizaje . Antecedentes y perspectivas. Revista Virtual Universidad Católica Del Norte, 1(38), 40-55.

Romero, L., Amo, A. De, Carmen, M., Borja, G., Romero, C. L., Alarcón, C., ... Borja, G. (2011). Adopción de redes sociales virtuales: ampliación del modelo de aceptación tecnológica integrando confianza y riesgo percibido. Cuadernos de Economía Y Dirección de La Empresa, 14(3), 194-205. http://doi.org/10.1016/j. cede.2010.12.003

Ruiz, T. (2015). La identidad asexual: de la masculinización social a las redes sociales virtuales. EMPIRIA. Revista de Metodología de Las Ciencias Sociales, (32), $129-167$.

Stornaiuolo, Amy; DiZio, Jennifer; Hellmich, E. D. (2013). Expanding Community : Youth, Social Networking, and Schools. Comunicar, 2O(40).

Suriá, \& Raquel. (2012). Redes sociales online y su utilización para mejorar las habilidades sociales en jóvenes con discapacidad. Escritos de Psicología - Psychological Writings, 5(3), 16-23. http://doi.org/10.5231/psy.writ.2012.1809

Vázquez, M. Y. G., Sexto, C. F., Rocha, Á., \& Aguilera, A. (2016). Mobile Phones and Psychosocial Therapies with Vulnerable People: a First State of the Art. Journal of Medical Systems, 40(6), 1-12.

Zornoza, A., Orengo, V., \& Peiró, J. (2008). Los procesos de influencia social en entornos virtuales y su incidencia sobre la eficacia grupal. Revista Latinoamericana de Psicología, 4O(2), 213-227. 